\title{
Solitary waves in a Hall solar wind plasma
}

\author{
I. Ballai ${ }^{1,2}$, J. C. Thelen ${ }^{2}$, and B. Roberts ${ }^{2}$ \\ ${ }^{1}$ Space and Atmosphere Research Center (SPARC), Department of Applied Mathematics, University of Sheffield, \\ Hounsfield Road, Hicks Building, Sheffield S3 7RH, UK \\ 2 School of Mathematics and Statistics, University of St Andrews, St Andrews, Fife, KY16 9SS, Scotland, UK
}

Received 2 April 2002 / Accepted 14 April 2003

\begin{abstract}
In the solar wind, the effect of the Hall current generated perpendicular to the ambient magnetic field can influence the plasma behaviour. In particular, the Hall current introduces wave dispersion which may compensate the nonlinear steepening of waves. In the presence of viscosity, these effects lead to a slowly decaying KdV soliton. Here we investigate a solitary wave propagating obliquely to an ambient magnetic field. Our results show that the nonlinear slow magnetoacoustic wave, that arises from nonlinear steepening, propagates faster than the linear slow mode speed; by contrast, the nonlinear fast magnetoacoustic wave propagates slower than the linear fast wave speed. We apply our calculations to the observed non-thermal Doppler broadening of the ion emission line, measured by the SoHO UVCS telescope in the acceleration region of the solar wind. The observed line broadening is recovered in our theory by the nonlinear slow magnetoacoustic waves; by contrast, fast magnetoacoustic produce too narrow broadening.
\end{abstract}

Key words. magnetohydrodynamics (MHD) - Sun: solar wind - waves

\section{Introduction}

Recent theoretical and observational developments have led to a degree of convergence in the understanding of the properties of the solar wind and the likely mechanisms responsible for its acceleration, especially through high-resolution observations made by the space satellites Ulysses and SoHO.

The solar wind can be regarded as the extension of the solar corona into interplanetary space. We can distinguish a fast component which originates from coronal holes and is being accelerated and a slow component which emanates from equatorial regions. At large distances from the Sun, the fast solar wind streams, which always have a unique polarity, exhibit relatively steady speeds of 700 to $800 \mathrm{~km} \mathrm{~s}^{-1}$, best demonstrated by Ulysses observations (see, e.g. Phillips et al. 1995; Woch et al. 1997). The slow component is denser than the fast one and at $1 \mathrm{AU}$ its speed is roughly $400 \mathrm{~km} \mathrm{~s}^{-1}$. The slow solar wind is much more complicated than the fast solar wind, since its properties vary on all time scales and it has a filamentary structure. Different source locations of the slow solar wind are under discussion: the edges of the polar coronal holes, the edges of streamer structures, or small loop structures in the equatorial region of the Sun. All these sources need magnetic reconnection processes to open up magnetic field lines and release plasma into space. It could well be that the slow solar wind emanates from several sources.

Send offprint requests to: I. Ballai, e-mail: i.ballai@sheffield.ac.uk
The theoretical treatment of the solar wind acceleration has been initiated by Parker (1958), based on the concept of an expansion of the hot $10^{6} \mathrm{~K}$ corona. It has become clear that the heating of the solar corona and the generation of the solar wind are closely related and that a distributed energy source is required in the inner corona to account for the basic features of the solar wind. Despite of many attempts to explain the acceleration of the solar wind in the principal acceleration region (between 1 and $5 R_{\odot}$ ), the exact mechanism responsible for this effect remains unknown; for a recent review see Antonucci (1999). Thermal conduction (e.g. Kopp \& Holzer 1976; Holzer \& Leer 1980; Davila 1985) is not sufficient to explain the observed flow speed of high-speed streams. Large amplitude Alfvén waves have been considered (e.g. Ofman \& Davila 1995; Smith et al. 1995; Grappin et al. 2000) but the gained momentum supply remains too low. The evidence of compressional waves in the solar wind shown by the CDS and UVCS telescopes onboard SoHO may suggest the idea that these waves may account for the required energy for plasma acceleration.

Chromospheric network activity, seen in low transition region lines, is a viable candidate for providing the energy and mass sources of the corona and solar wind (Axford \& McKenzie 1992; Axford et al. 1999; De Pontieu et al. 2003) but direct evidence is lacking.

Solitons and solitary waves are known to occur in many laboratory and terrestrial circumstances and there are theoretical reasons to expect their occurrence is space plasmas. However, the existing spatial and temporal resolution of satellite 
instruments are not sufficient to provide direct evidence for their existence. Nevertheless, some theoretical attempts have been made to explain solar (see, for example, Roberts \& Mangeney 1982; Roberts 1985, 1987; Edwin \& Roberts 1986; Hollweg \& Roberts 1984) and solar wind (see, for example, Mjolhus 1976; Spangler \& Sheerin 1982; Spangler 1985; Dawson \& Fontán 1988, 1990; Mjolhus \& Hada 1997; Ofman \& Davila 1997; Nakariakov \& Roberts 1999) phenomena by means of solitons.

Solitons are finite-amplitude waves of permanent form which owe their existence to a balance between nonlinear wave steepening and linear wave dispersion. Here we suppose that there is enough amount of energy at the dispersive scale to have large amplitude solitons. Typically, they consist of a single isolated wave whose speed is an increasing function of amplitude. Nonlinearity is often a consequence of large spatial and temporal scales and is very likely to occur in astrophysical plasmas. Dispersion, on the other hand, can arise from an existing wave guide (plasma structuring), sometimes called geometrical dispersion, or from plasma effects (physical dispersion) introduced by, for example, the generalized Ohm's law. In general, these two effects give rise to different dispersive behaviours but they have the same general result: the creation of a length scale in addition to the natural length scale (their wavelength) of the waves. Here, we consider the dispersion caused by plasma effects through the Hall term present in the generalized Ohm's law.

The study of compressional magnetohydrodynamic waves in Hall plasmas is a relatively new subject (see, for example, Ruderman 1987; Ghanashev et al. 1996; Zhelyazkov et al. 1996; Zhelyazkov \& Mann 1999; Ruderman 2002; Miteva et al. 2003). As stressed by Huba (1995), Hall MHD is relevant to plasma dynamics occurring on length scales shorter than an ion inertial length, $c / \omega_{i}$, where $c$ is the speed of light and $\omega_{i}$ is the ion plasma frequency. Inclusion of the Hall term in the magnetohydrodynamic induction equation is known to affect the polarization of waves, because it includes the dispersion of Alfvén waves near the ion cyclotron frequency.

Observational evidence of compressional waves in the solar wind has recently been obtained. DeForest \& Gurman (1998), using high-cadence EIT/SoHO observations, indicated some quasi periodic fluctuations with periods of $10-15 \mathrm{~min}$ in solar plumes, with a filamentary structure within the plume on a spatial scale of 3-5 arcsec. These fluctuations were identified as sound or slow magnetoacoustic waves propagating along the plumes at speeds of $75-150 \mathrm{~km} \mathrm{~s}^{-1}$. Ofman et al. (2000) and Banerjee et al. (2001) detected quasi periodic variations in the polarization brightness at $1.9 R_{\odot}$, in both plume and interplume regions. Their Fourier power spectrum shows significant peaks around $1.6-2.5 \mathrm{mHz}$, and additional smaller peaks at longer and shorter time-scales. Their wavelet analysis of the polarization brightness time series shows that the coherence time of the fluctuations is about $30 \mathrm{~min}$. Recently, Banerjee et al. (2001) used CDS/SoHO images to detect long period slow magnetoacoustic waves in the solar inter-plume region, the waves being produced at network boundaries in the coronal hole.
Here we follow the idea of Ofman \& Davila (1997) and suppose that the Doppler broadening of the ion emission line observed in the UVCS telescope is attributable to nonlinear solitary waves propagating in the solar corona. Using the propagation speed of a nonlinear slow magnetoacoustic wave soliton, which is slowly decaying because of viscosity, we show that the calculated Doppler broadening is consistent with UVCS observations.

The paper is organized as follows: in Sect. 2 the basic equations are introduced and the main assumptions discussed. The derivation of the Korteweg-deVries-Burgers equation is presented in Sect. 3. Section 4 is devoted to an application of this nonlinear evolution equation as an explanation of the observed Doppler-broadening of the ion emission line observed in the solar wind. Finally, in Sect. 5 we summarise our results.

\section{Basic equations}

We model the solar wind by a viscous plasma penetrated by a uniform magnetic field. The magnetic field is unidirectional and situated in the $x z$-plane: $\boldsymbol{B}_{0}=\left(B_{0} \sin \alpha\right) \hat{\boldsymbol{x}}+B_{0}(\cos \alpha) \hat{\boldsymbol{z}}$, where $B_{0}$ is the field strength, $\alpha$ is the angle between the magnetic field and the $z$-axis, and $\hat{\boldsymbol{x}}, \hat{z}$ are unit vectors.

The Hall term appears in the generalized Ohm's law as an effect of a generated current (Hall current) perpendicular to the ambient magnetic field. This effect is produced by charged particles drifting across the magnetic field. The presence of this term introduces a new length scale (the ion inertial length) which renders wave propagation as dispersive.

We suppose that the plasma is collision dominated, appropriate for the solar wind in regions close to the Sun. The plasma motion is described in the Hall-MHD approximation (see, for example, Zhelyazkov et al. 1996):

$$
\begin{aligned}
& \frac{D \rho}{D t}+\rho \nabla \cdot \boldsymbol{v}=0, \\
& \rho \frac{D \boldsymbol{v}}{D t}=-\nabla p+\frac{1}{\mu}[(\nabla \times \boldsymbol{B}) \times \boldsymbol{B}]+v \rho[\nabla \\
& \frac{\partial \boldsymbol{B}}{\partial t}-\nabla \times(\boldsymbol{v} \times \boldsymbol{B})=\frac{m_{\mathrm{i}}}{e \rho \mu}(\boldsymbol{B} \cdot \nabla) \nabla \times \boldsymbol{B}, \\
& \frac{D}{D t}\left(\frac{p}{\rho^{\gamma}}\right)=0,
\end{aligned}
$$$$
\rho \frac{D \boldsymbol{v}}{D t}=-\nabla p+\frac{1}{\mu}[(\nabla \times \boldsymbol{B}) \times \boldsymbol{B}]+v \rho\left[\nabla^{2} \boldsymbol{v}+\frac{1}{3} \nabla(\nabla \cdot v)\right],
$$

where $D / D t=\partial / \partial t+\boldsymbol{v} \cdot \nabla$ is the advective operator, $\boldsymbol{v}$ is the velocity, $p$ and $\rho$ are the pressure and density, $v$ is the coefficient of kinematic viscosity, $\gamma$ is the adiabatic index, $m_{\mathrm{i}}$ is the ion mass, and $e$ is the elementary charge on an electron. The magnetic induction vector is $\boldsymbol{B}$, satisfying $\nabla \cdot \boldsymbol{B}=0$. The perturbed magnetic field is $\boldsymbol{b}=\left(b_{x}, b_{y}, b_{z}\right)$ and the flow is $\boldsymbol{v}=\left(v_{x}, v_{y}, v_{z}\right)$. The Hall character of the plasma is reflected by the term involving $m_{i}$ in Eq. (3).

We consider small but finite amplitude perturbations about a uniform equilibrium, writing all physical quantities $f$ in the form

$f=f_{0}+\tilde{f}$, 
where $f_{0}$ is an equilibrium value and $\tilde{f}$ its Eulerian perturbation. The tilde will be omitted subsequently. We suppose wave propagation to be along the $z$-axis, and we choose $\partial / \partial x=$ $\partial / \partial y=0$.

The system of MHD Eqs. (1)-(4) can be written as

$\frac{\partial b_{x}}{\partial t}+B_{0} \frac{\partial}{\partial z}\left(v_{z} \sin \alpha-v_{x} \cos \alpha\right)=N_{1}+H_{x}$,

$\frac{\partial b_{y}}{\partial t}-B_{0} \cos \alpha \frac{\partial v_{y}}{\partial z}=N_{2}+H_{y}$,

$\frac{\partial b_{z}}{\partial t}=0$

$\rho_{0} \frac{\partial v_{x}}{\partial t}-\frac{B_{0} \cos \alpha}{\mu} \frac{\partial b_{x}}{\partial z}=N_{4}+D_{1}$

$\rho_{0} \frac{\partial v_{y}}{\partial t}-\frac{B_{0} \cos \alpha}{\mu} \frac{\partial b_{y}}{\partial z}=N_{5}+D_{2}$,

$\rho_{0} \frac{\partial v_{z}}{\partial t}+\frac{\partial p}{\partial z}+\frac{B_{0} \sin \alpha}{\mu} \frac{\partial b_{x}}{\partial z}=N_{6}+D_{3}$,

$\rho_{0} \frac{\partial p}{\partial t}-\rho_{0} c_{\mathrm{S}}^{2} \frac{\partial \rho}{\partial t}=N_{7}$,

$\frac{\partial \rho}{\partial t}+\rho_{0} \frac{\partial v_{z}}{\partial z}=N_{8}$

where $N_{i}(i=1 \ldots 8)$ are the nonlinear terms (with $N_{3} \equiv 0$ ), $H_{x}$ and $H_{y}$ are the projections of the Hall term on the $x$ and $y$ axis, and $D_{1}, D_{2}$ and $D_{3}$ are the dissipative terms. According to Eq. (7) we choose $b_{z}=0$.

Restricting our analysis to quadratic nonlinearities only, the nonlinear terms are

$N_{1}=-\frac{\partial}{\partial z}\left(v_{z} b_{x}\right), \quad N_{2}=-\frac{\partial}{\partial z}\left(v_{z} b_{y}\right)$,

$N_{4}=-\rho \frac{\partial v_{x}}{\partial t}-\rho_{0} v_{z} \frac{\partial v_{x}}{\partial z}, \quad N_{5}=-\rho \frac{\partial v_{y}}{\partial t}-\rho_{0} v_{z} \frac{\partial v_{y}}{\partial z}$,

$N_{6}=-\rho \frac{\partial v_{z}}{\partial t}-\rho_{0} v_{z} \frac{\partial v_{z}}{\partial z}-\frac{b_{x}}{\mu} \frac{\partial b_{x}}{\partial z}-\frac{b_{y}}{\mu} \frac{\partial b_{y}}{\partial z}$,

$N_{7}=-\rho \frac{\partial p}{\partial t}-\rho_{0} v_{z} \frac{\partial p}{\partial z}+\gamma p \frac{\partial \rho}{\partial t}+\gamma p_{0} w \frac{\partial \rho}{\partial z}$,

$N_{8}=-\frac{\partial}{\partial z}\left(\rho v_{z}\right)$.

The two components of the Hall term are

$H_{x}=-\frac{v_{\mathrm{A}}^{2}}{\omega_{\mathrm{i}}} \cos \alpha \frac{\partial^{2} b_{y}}{\partial z^{2}}, \quad H_{y}=\frac{v_{\mathrm{A}}^{2}}{\omega_{\mathrm{i}}} \cos \alpha \frac{\partial^{2} b_{x}}{\partial z^{2}}$,

where $\omega_{\mathrm{i}}=e B_{0} / m_{\mathrm{i}}$ is the ion-cyclotron frequency.

The components of the dissipative term can be written as

$D_{1}=v \rho \frac{\partial^{2} v_{x}}{\partial z^{2}}, \quad D_{2}=v \rho \frac{\partial^{2} v_{y}}{\partial z^{2}}, \quad D_{3}=\frac{4}{3} v \rho \frac{\partial^{2} v_{z}}{\partial z^{2}}$.

The system of Eqs. (5)-(19) will be used in the next section to derive the nonlinear equation that describes the evolution of a compressible perturbation in a Hall plasma.

\section{Derivation of the nonlinear evolutionary equation}

To derive the governing equation we use the standard method of multiple scaling. We suppose that the nonlinearity, the dispersive effects caused by the presence of the Hall term and the effect of viscous damping are all weak and are of the same order. We are then able to introduce the stretched variables $\xi$ and $\tau$ through

$\xi=\epsilon^{1 / 2}(z-V t), \quad \tau=\epsilon^{3 / 2} t$,

where $V$ is the phase velocity of a wave (slow or fast magnetoacoustic), $\epsilon$ is a small parameter measuring the amplitude of the waves, and $\tau$ is the "slow" time describing the wave evolution in a coordinate system moving with the wave. Perturbations $f$ are expanded in series as

$f=\epsilon f_{1}+\epsilon^{2} f_{2}+\ldots$

for the sound variables $\rho, p, v_{x}, v_{z}$ and $b_{x}$, and

$g=\epsilon^{1 / 2}\left(\epsilon g_{1}+\epsilon^{2} g_{2}+\ldots\right)$

for the Alfvénic variables $v_{y}$ and $b_{y}$. The two expansions show that the first non-vanishing term in the sound variables expansion is $\epsilon^{-1 / 2}$ larger than the first non-vanishing term in the expansion of the Alfvénic perturbations. In order that the viscosity is of the same order as the nonlinearity and dispersion, we re-scale the coefficient of viscosity $v$ as

$v=\epsilon^{3 / 2} \bar{v}$.

Substituting the stretched variables (20) and the perturbation expansions (21) and (22) into the system of Eqs. (5)-(18) and equating powers of $\epsilon$, we obtain a sequence of equations for the variables $f_{i}$ and $g_{i}$. All variables (at the lowest order, $O\left(\epsilon^{3 / 2}\right)$ ) can be expressed in terms of the perturbed density, $\rho_{1}$ :

$v_{x 1}=-\frac{V^{2}-c_{\mathrm{S}}^{2}}{V}(\cot \alpha) \frac{\rho_{1}}{\rho_{0}}, \quad p_{1}=c_{\mathrm{S}}^{2} \rho_{1}$,

$b_{x 1}=B_{0} \frac{V^{2}-c_{\mathrm{S}}^{2}}{v_{\mathrm{A}}^{2}}(\sec \alpha) \frac{\rho_{1}}{\rho_{0}}, \quad v_{z 1}=V \frac{\rho_{1}}{\rho_{0}}$,

where the wave speed $V$ satisfies the linear dispersion relation $V^{4}-V^{2}\left(c_{\mathrm{S}}^{2}+v_{\mathrm{A}}^{2}\right)+v_{\mathrm{A}}^{2} c_{\mathrm{S}}^{2} \cos ^{2} \alpha=0 ;$

$v_{\mathrm{A}}^{2}=B_{0}^{2} / \mu \rho_{0}$ and $c_{\mathrm{S}}^{2}=\gamma p_{0} / \rho_{0}$ are the squares of the Alfvén and sound speed, respectively. Relation (24) means that the fast and slow magnetoacoustic waves will propagate with a phase speed given by

$V_{\mathrm{f}, \mathrm{s}}^{2}=\frac{1}{2}\left(c_{\mathrm{S}}^{2}+v_{\mathrm{A}}^{2} \pm \sqrt{c_{\mathrm{S}}^{4}+v_{\mathrm{A}}^{4}-2 c_{\mathrm{S}}^{2} v_{\mathrm{A}}^{2} \cos 2 \alpha}\right)$,

where the indices "f" and "s" denote the phase speed of fast and slow magnetoacoustic waves, respectively. The $y$ component of the induction and momentum equations gives (to the lowest order)

$v_{y}=\frac{1}{\omega_{\mathrm{i}}}\left(\frac{V^{2}-c_{\mathrm{S}}^{2}}{V^{2}-v_{\mathrm{A}}^{2} \cos ^{2} \alpha}\right) v_{\mathrm{A}}^{2} \cos ^{2} \alpha \frac{\partial}{\partial \xi}\left(\frac{\rho_{1}}{\rho_{0}}\right)$, 
$b_{y 1}=-\frac{1}{\omega_{\mathrm{i}}}\left(\frac{V^{2}-c_{\mathrm{S}}^{2}}{V^{2}-v_{\mathrm{A}}^{2} \cos ^{2} \alpha}\right) B_{0} V \cot \alpha \frac{\partial}{\partial \xi}\left(\frac{\rho_{1}}{\rho_{0}}\right)$.

Having in mind the expression of the Hall terms and their role in the process of wave propagation, we can say that the dispersion of the wave is generated by shear Alfvén waves through the Hall current.

In the second order approximation we obtain a system of equation for variables with index " 2 ". These quantities are expressed in terms of the variables obtained in the first order of approximation. Eliminating variables, we obtain

$$
\frac{\partial \rho_{1}}{\partial \tau}+\alpha_{1} \rho_{1} \frac{\partial \rho_{1}}{\partial \xi}-\alpha_{2} \frac{\partial^{3} \rho_{1}}{\partial \xi^{3}}-\alpha_{3} \frac{\partial^{2} \rho_{1}}{\partial \xi^{2}}=0 .
$$

Equation (27) is the main result of this paper. It is the Korteweg-deVries-Burgers equation which here describes the evolution of a density disturbance in a viscous plasma with the condition that $\rho_{1}$ vanishes at $|\xi| \rightarrow \infty$. The coefficients $\alpha_{1}, \alpha_{2}$ and $\alpha_{3}$ are given by

$$
\begin{aligned}
& \alpha_{1}=\frac{V\left[c_{\mathrm{S}}^{2}(\gamma+1)\left(V^{2}-v_{\mathrm{A}}^{2} \cos ^{2} \alpha\right)+3 V^{2}\left(V^{2}-c_{\mathrm{S}}^{2}\right)\right]}{2 \rho_{0}\left(2 V^{4}-v_{\mathrm{A}}^{2} c_{\mathrm{S}}^{2} \cos ^{2} \alpha\right)}, \\
& \alpha_{2}=\frac{v_{\mathrm{A}}^{4} \cos ^{2} \alpha V^{3}\left(V^{2}-c_{\mathrm{S}}^{2}\right)}{2 \omega_{\mathrm{i}}^{2}\left(V^{2}-v_{\mathrm{A}}^{2} \cos ^{2} \alpha\right)\left(2 V^{4}-v_{\mathrm{A}}^{2} c_{\mathrm{S}}^{2} \cos ^{2} \alpha\right)}, \\
& \alpha_{3}=\frac{v\left(4 V^{4}-V^{2} v_{\mathrm{A}}^{2} \cos ^{2} \alpha-3 v_{\mathrm{A}}^{2} c_{\mathrm{S}}^{2} \cos ^{2} \alpha\right)}{6\left(2 V^{4}-v_{\mathrm{A}}^{2} c_{\mathrm{S}}^{2} \cos ^{2} \alpha\right)} .
\end{aligned}
$$

Equation (27) describes the nonlinear evolution of both slow and fast magnetoacoustic waves in a dissipative plasma, but not their coupling. The coefficients $\alpha_{1}, \alpha_{2}$ and $\alpha_{3}$ depend on the angle $\alpha$ of propagation and the plasma beta, defined as $\beta=2 c_{\mathrm{S}}^{2} /\left(\gamma v_{\mathrm{A}}^{2}\right) ; \beta$ is the ratio of the kinetic and magnetic pressures. In the solar wind near the Sun, $\beta$ is small; we confine attention to this case, considering three specific values, namely $\beta=1.2 \times 10^{-3}, 1.2 \times 10^{-2}$ and $6 \times 10^{-2}$. The propagation angle $\alpha$ varies between 0 (parallel propagation) and $\pi / 2$ (perpendicular propagation).

Consider first fast magnetoacoustic waves. In this case, the nonlinear parameter $\alpha_{1} \rho_{0} / c_{\mathrm{S}}$ shows a strong dependence on $\beta$ (see Fig. 1). For a fixed value of $\beta$, this parameter is practically independent of the propagation angle. In fact, the parameter shows a slight change with respect to $\alpha$; to show this, we magnified the dependence of this parameter of the propagation angle for the case $\beta=1.2 \times 10^{-2}$ (see Fig. 2). By contrast, the dispersive parameter $\alpha_{2} \omega_{\mathrm{i}}^{2} / c_{\mathrm{S}}^{3}$ shows a very steep variation only for small values of the propagation angle corresponding to almost parallel propagation; see Fig. 3. As the angle of propagation increases, the coefficient $\alpha_{2} \omega_{\mathrm{i}}^{2} / c_{\mathrm{S}}^{3}$ saturates and tends to zero, i.e. for perpendicular propagation, waves are no longer dispersive. In this extreme the wave evolution is described by Burgers equation and the solution of this equation is a fast shock wave, propagating perpendicular to the ambient magnetic field, whose amplitude decays due to viscosity and the wavefront steepens due to nonlinearity (for a detailed discussion, see Adam 1975). For propagation parallel to the field

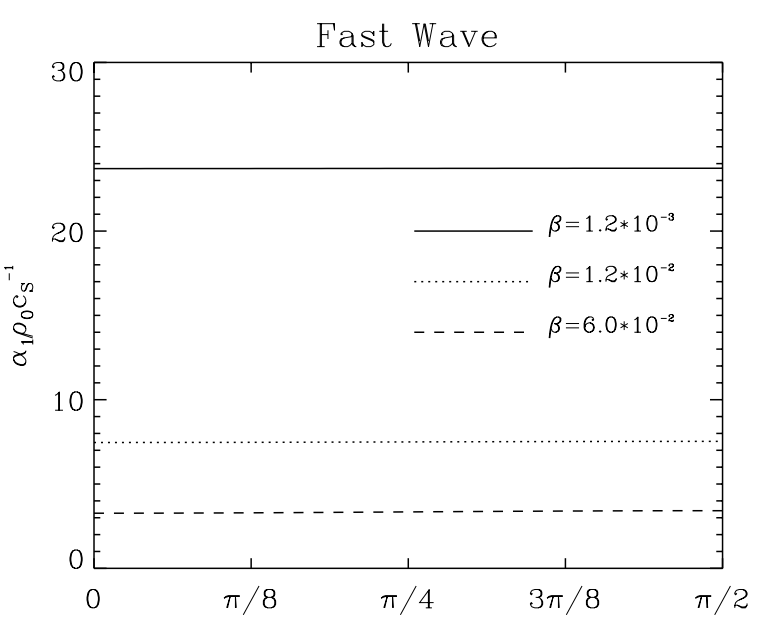

Fig. 1. The variation of the nonlinear parameter $\alpha_{1} \rho_{0} / c_{\mathrm{S}}$ in the fast magnetoacoustic wave as a function of propagation angle $\alpha$, for small plasma- $\beta$.

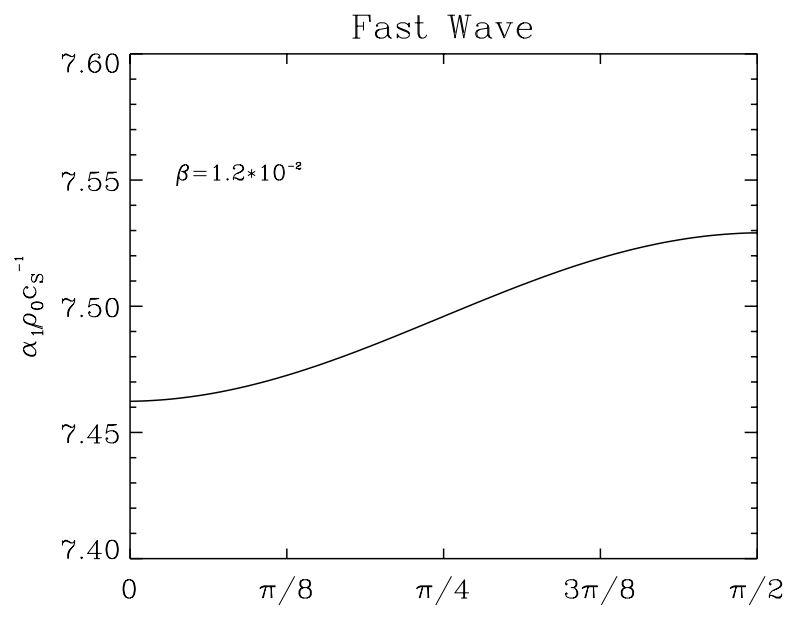

Fig. 2. The variation of the nonlinear parameter $\alpha_{1} \rho_{0} / c_{\mathrm{S}}$ in the fast magnetoacoustic wave as a function of propagation angle $\alpha$, for $\beta=$ $1.2 \times 10^{-2}$.

( $\alpha=0$ ), the evolutionary equation shows a degeneracy (the dispersive parameter tends to infinity) and the evolution of a nonlinear dispersive fast magnetoacoustic wave is described by the derivative nonlinear Schrödinger (DNLS) equation (Mjølhus 1976; Mjølhus \& Hada 1997). Finally, the parameter of the dissipative term, $\alpha_{3} / \bar{v}$, increases with propagation angle but is almost independent of the plasma- $\beta$. The damping parameter $\alpha_{3} / \bar{v}$ is at a maximum perpendicular to the equilibrium magnetic field; see Fig. 4.

Turning now to the slow magnetoacoustic waves, the nonlinearity parameter $\alpha_{1} \rho_{0} / c_{\mathrm{S}}$ decreases with $\alpha$ and for propagation angles close to the perpendicular the parameter is independent of the plasma- $\beta$; see Fig. 5. Since slow wave propagation is not permitted across the field, $\alpha_{1}=0$ at $\alpha=\pi / 2$. The coefficient of the dispersive term, $\alpha_{2} \omega_{\mathrm{i}}^{2} / c_{\mathrm{S}}^{3}$, is negative for $0<\alpha<\pi / 2$ and for all $\beta$ (see Fig. 6). As with fast magnetoacoustic waves, for propagation parallel to the equilibrium magnetic field $(\alpha=0)$ waves are no longer dispersive and their nonlinear evolution is described by Burgers equation, with a solution in the form of slowly decaying slow shock waves. 


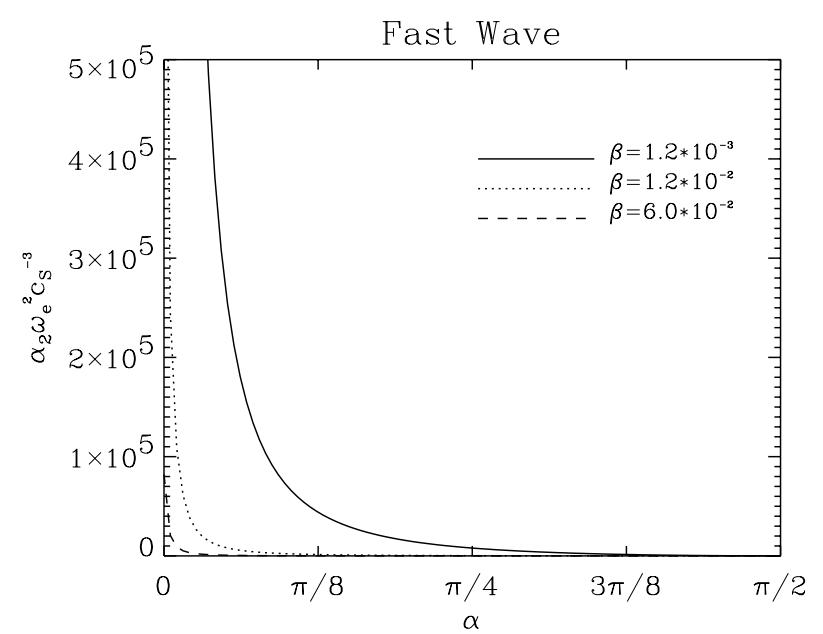

Fig. 3. The variation of the dispersive parameter $\alpha_{2} \omega_{\mathrm{i}}^{2} / c_{\mathrm{S}}^{3}$ in the fast magnetoacoustic wave as a function of propagation angle $\alpha$ for different values of plasma- $\beta$.

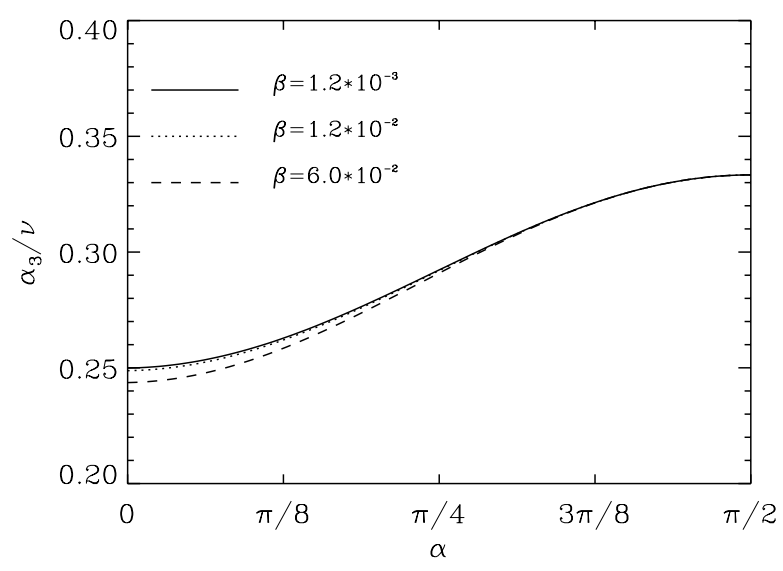

Fig. 4. The variation of the viscous parameter, $\alpha_{3} / v$, in the fast magnetoacoustic wave as a function of the propagation angle, $\alpha$, for small plasma- $\beta$.

The parameter $\alpha_{3}$ of the dissipative term shows a slowly decreasing tendency with respect to the propagation angle. Its value (similar to the case of fast waves) does not change significantly with respect to $\beta$; see Fig. 7. The damping parameter is at a maximum for propagation parallel to the equilibrium magnetic field.

Equation (27) has an approximate solitary wave solution, which in the absence of dissipation would be a soliton. In the frame of reference moving with the wave, this solution is

$\rho=\tilde{\rho}\left(1+\frac{\tau}{t_{\mathrm{d}}}\right)^{-1} \operatorname{sech}^{2}\left(\frac{\xi-\theta \tau}{L}\right)$,

where

$L=\sqrt{\frac{12\left|\alpha_{2}\right|}{\tilde{\rho} \alpha_{1}}}, \quad \theta=\frac{\tilde{\rho} \alpha_{1}}{3}, \quad t_{\mathrm{d}}=\frac{15\left|\alpha_{2}\right|}{16 \tilde{\rho} \alpha_{1} \alpha_{3}}$

provides the length $L$, speed (in the moving frame of reference), and the viscous decay time $t_{\mathrm{d}}$ of the nonlinear wave. The constant $\tilde{\rho}$ is the amplitude of the soliton-like wave; the width of the soliton decreases with increasing amplitude, following a

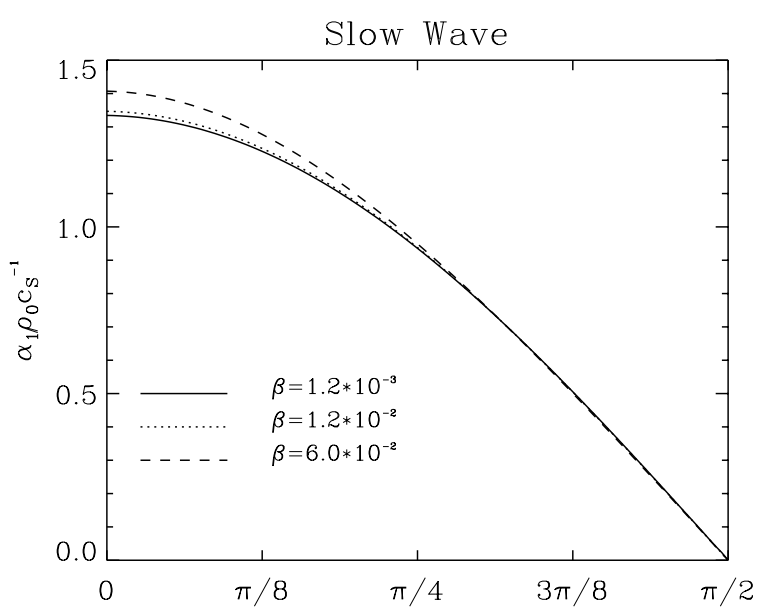

Fig. 5. The variation of the nonlinear parameter, $\alpha_{1} \rho_{0} / c_{\mathrm{S}}$, in the slow magnetoacoustic wave as a function of the propagation angle $\alpha$, for small plasma- $\beta$.

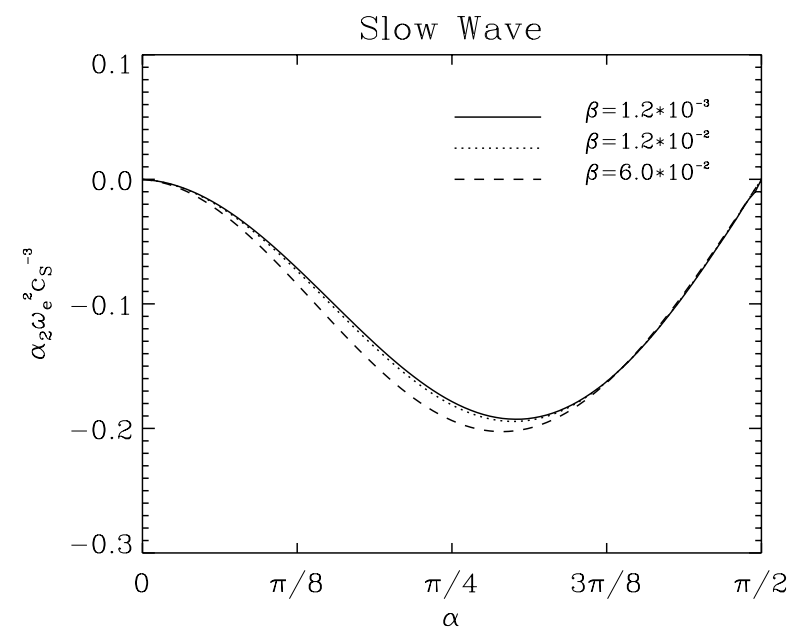

Fig. 6. The variation of the dispersive parameter, $\alpha_{2} \omega_{\mathrm{i}}^{2} / c_{\mathrm{S}}^{3}$, in the slow magnetoacoustic wave as a function of the propagation angle $\alpha$, for small plasma- $\beta$.

$\tilde{\rho}^{-1 / 2}$ law. As we go further from the Sun, the density decreases and so the width of this soliton increases. The effect of viscosity is to induce a slow decay of the solitary wave.

\section{Application}

Consider first solitary waves that arise from slow magnetoacoustic modes; for such modes, $\alpha_{1}>0, \alpha_{3}>0$ and $\alpha_{2}<0$. Accordingly, the decaying soliton solution (29) gives a propagation speed (in the original coordinate system) of

$V_{\mathrm{s}}+\frac{1}{3} \tilde{\rho} \alpha_{1}$

showing an increase in speed (above the linear wave speed $V_{\mathrm{s}}$ of a slow magnetoacoustic wave) with increasing amplitude $\tilde{\rho}(>0)$. This is positive dispersion: waves with larger wavelengths propagate faster than waves with shorter wavelengths.

Thus, when the conditions of formation of a solitary wave are satisfied (the steepening due to nonlinearity is balanced by the broadening due to dispersion), a solitary wave appears 


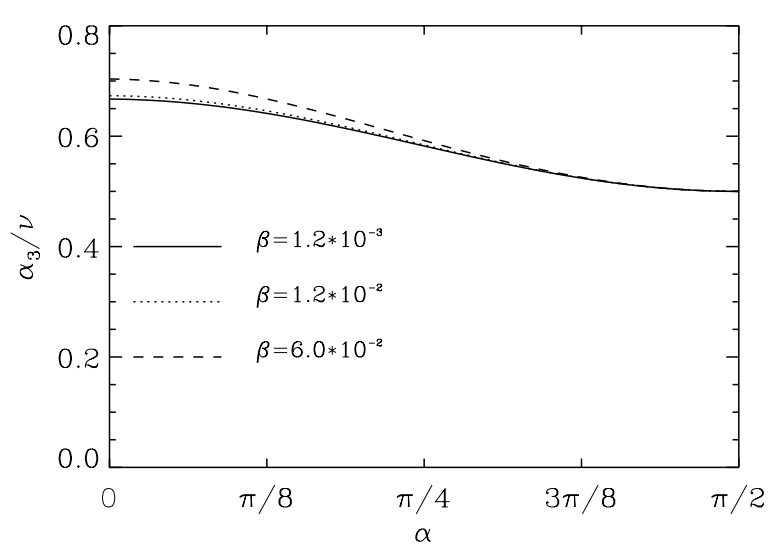

Fig. 7. The variation of the viscous parameter, $\alpha_{3} / v$, in the slow magnetoacoustic wave as a function of the propagation angle $\alpha$, for small plasma- $\beta$.

which moves faster than the wave speed of the corresponding linear wave. From Fig. 5, we see that the addition to the linear propagation speed is below the Alfvén speed.

Consider now solitary waves which in the linear limit propagate as fast magnetoacoustic waves; here $\alpha_{1}>0, \alpha_{3}>0$ and $\alpha_{2}>0$. In this case, the propagation speed of the solitary wave (in the original coordinate system) is

$V_{\mathrm{f}}-\frac{1}{3} \tilde{\rho} \alpha_{1}$

giving a speed which is smaller than the propagation speed of the corresponding linear fast magnetoacoustic wave. These are waves with negative dispersion: waves with shorter wavelengths propagate faster than those with longer wavelengths.

We compare our theoretical results with the observational results found by the Ultra Violet Coronograph Spectrometer (UVCS) on board the SoHO satellite. The aim of this instrument is to study electron and ion temperatures between $1.3 R_{\odot}$ and $10 R_{\odot}$, with high resolution spectrometers, to locate and characterize the coronal source regions of the solar wind and to understand how the solar wind is accelerated. Kohl et al. (1996) and Noci et al. (1996) have reported an ion emission line that is Doppler-shifted by $300 \mathrm{~km} \mathrm{~s}^{-1}$, corresponding to unresolved motions at heliocentric distance $1.7 R_{\odot}$.

The unresolved non-thermal motions (e.g. wave motions) may also contribute to the width of the emission lines of the ion spectra. The kinetic temperature, $T_{\mathrm{k}}$, of a certain ion is given by

$T_{\mathrm{k}}=\frac{m_{\mathrm{i}} v_{\mathrm{d}}^{2}}{2 k_{\mathrm{B}}}=T_{\text {ion }}+\frac{m_{\mathrm{i}} \zeta^{2}}{2 k_{\mathrm{B}}}$,

where $m_{\mathrm{i}}$ is the ion mass, $k_{\mathrm{B}}$ is the Boltzmann constant, and $v_{\mathrm{d}}$ is the velocity of the ions determined from the half-width at $(1 / e) I_{0}$ of the Doppler-broadened emission line. Here $I_{0}$ is the peak intensity of the line, $T_{\text {ion }}$ is the ion temperature and $\zeta$ is the bulk velocity along the line of sight, due to processes such as waves, turbulence, non-radial coronal expansion, etc. UVCS measurements suggest that the observed Doppler broadening is a result of thermal and non-thermal motions that have the same magnitude for $\mathrm{H} \mathrm{I}$ and $\mathrm{O}$ VI ions and is about $300 \mathrm{~km} \mathrm{~s}^{-1}$. The fact that the Doppler-broadening is independent of the ion mass

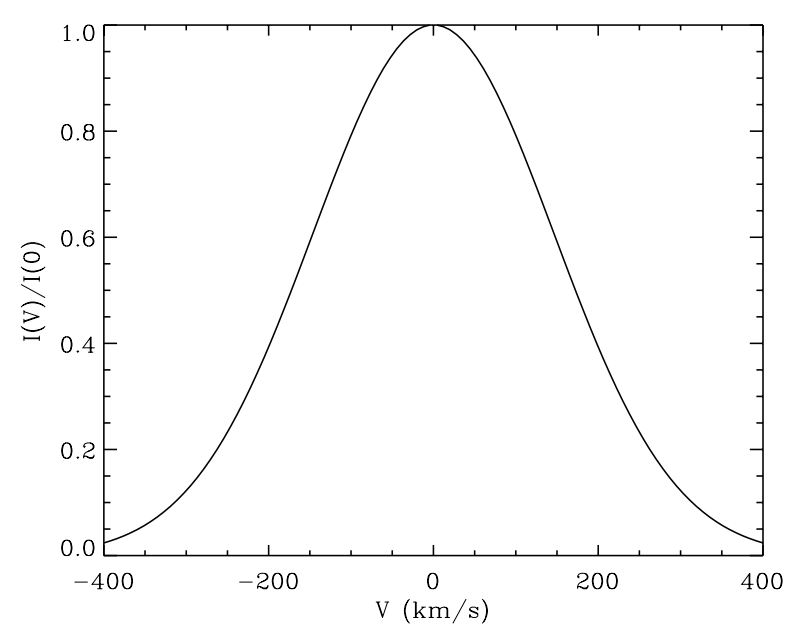

Fig. 8. Doppler broadening due to unresolved wave motions. The intensity fluctuations $I(v) / I_{0}$ determined by Eq. (31) are calculated for motions $v=v_{y}$, the component of the velocity in the line of sight direction, in the slow magnetoacoustic soliton wave. The density $\rho(t)$ is given by (29), calculated at the "observation point" $z=1.7 R_{\odot}$ taking $\alpha=9^{\circ}$ and $\beta=5 \times 10^{-2}$.

suggests that the observed motions may be due to the effect of unresolved wave motions integrated over several hours of observation.

Now, the proton temperature at $1.7 R_{\odot}$ is about $2.5 \times 10^{6} \mathrm{~K}$ (Antonucci 1999), which corresponds to a thermal velocity $v_{\text {th }}$ of $207 \mathrm{~km} \mathrm{~s}^{-1}$. The distribution $I(v)$ of a line intensity in terms of a velocity, $v$, due to a thermal motion is obtained by integrating the thermal emission shifted by the non-thermal wave velocity, $v_{\mathrm{w}}(t)$, in the line of sight (in our case $v_{y}$, the $y$-component of the velocity given by Eq. (26)), and is given by

$\frac{I(v)}{I_{0}}=\int_{t_{1}}^{t_{2}} \exp \left[-\left(\frac{v-v_{\mathrm{w}}(t)}{v_{\mathrm{th}}}\right)^{2}\right] \rho^{2}(t) \mathrm{d} t$,

where $I_{0}$ is the maximal intensity and $t_{1}$ and $t_{2}$ are the start and end observing times. The intensity of the line is weighted by $\rho^{2}$ to model the line emission dependence on density. The integration is taken over times longer that the period of the waves and it is about 4 hours, which is of the order of the UVCS integration time. Cranmer et al. (1999) proposed an empirical model of the polar coronal hole at solar minimum based on current observational results. One of their conclusions was that the plasma flow is not strictly radial, but is oblique. Based on their results, we suppose that the plasma flow makes an angle of $9^{\circ}$ with the vertical.

The result of the integration (31) for $\rho(t)$ given by (29) with $\alpha=9^{\circ}$, calculated at the "observation point" $z=1.7 R_{\odot}$, is given in Fig. 8 for the slow nonlinear magnetoacoustic wave; the shape of the model line profile is very close to Gaussian. The fast nonlinear wave produces a narrower $I(v)$, making it less able to match the observed broadening of $300 \mathrm{~km} \mathrm{~s}^{-1}$. The amplitude of the soliton has been calculated taking into account the value of the number density at the base of the coronal hole and the variation of the number density with respect to the heliocentric distances (see, e.g. Wurz \& Gabriel 1999). 
Thus the slow wave gives a profile width of $293 \mathrm{~km} \mathrm{~s}^{-1}$ (which corresponds to a kinetic temperature of $5 \times 10^{6} \mathrm{~K}$ ) in good agreement with the UVCS spectrometer observations (see Antonucci 1999; Cranmer et al. 1999, and references therein). The calculated width decreases with decreasing $\alpha$ and increases slightly for propagation angles larger than the $9^{\circ}$ we have chosen. Comparing the damping times for nonlinear slow and fast waves (the damping time is given by (30) for the same equilibrium parameters and for the propagation angle considered before, we obtain that the damping time of fast waves is approximately $10^{5}$ times larger than the corresponding damping time for slow waves, i.e. solitons arising from nonlinear steepening of slow magnetoacoustic waves are able to transfer their energy and momentum more effectively

\section{Conclusions}

In the theoretical model presented in this paper we have derived the Korteweg-deVries-Burgers equation as a description of solitary waves in a viscous magnetised Hall plasma, and applied that equation to explain the observed Doppler broadening in the emission line at $1.7 R_{\odot}$, measured in the solar wind. The presence of the Hall current perpendicular to the equilibrium magnetic field induces a dispersive effect which balances the nonlinear steepening of the wave. In particular, solutions of the Korteweg-deVries-Burgers equation in the form of travelling solitary waves are considered; the speed of the nonlinear wave varies linearly with the wave amplitude and wavelengths vary inversely as the square root of wave amplitude. We supposed that the observed non-thermal Doppler broadening of the ion emission line is due to unresolved (linear and nonlinear) slow MHD waves. The calculated Doppler broadening of the emission line, determined by using decaying KdV slow mode solitons, is in good agreement with the observed amplitude of Doppler-broadening in $\mathrm{HI}$ and O VI ions recorded by UVCS at $1.7 R_{\odot}$.

\section{References}

Adam, J. A., Ap\&SS, 36, 479

Antonucci, E. 1999, in 8th SoHO Workshop, ed. J. C. Vial, \& B. Kaldeich-Schürmann, ESA SP-46, 53

Axford, E., \& McKenzie, J. F. 1992, in Solar Wind VII, ed. E. Marsch, \& R. Schwenn (New York: Pergamon Press), 1

Axford, E., McKenzie, J. F., Sukhorukova, G. V., et al. 1999, Space Sci. Rev., 87, 25

Banerjee, D., O’Shea, E., Doyle, J. G., \& Goossens, M. 2001, A\&A, 377,691
Cranmer, S. R., Kohl, J. L., Noci, G., et al. 1999, ApJ, 511, 481

Davila, J. M. 1985, ApJ, 291, 328

Dawson, S. P., \& Fontán, C. F. 1988, Phys. Fluids, 31, 83

Dawson, S. P., \& Fontán, C. F. 1990, ApJ, 348, 761

DeForest, C. E., \& Gurman, J. B. 1998, ApJ, 501, L217

De Pontieu, B., Tarbell, T., \& Erdélyi, R. 2003, ApJ, in press [DOI : $10.1086 / 374928]$

Edwin, P. M., \& Roberts, B. 1986, Wave Motion, 8, 151

Ghanashev, I., Zhelyazkov, I., \& Stoykov, V. 1996, Phys. Plasmas, 3, 3540

Grappin, R., Léorat, J., \& Buttighoffer, A. 2000, A\&A, 362, 342

Hollweg, J. V., \& Roberts, B. 1985, J. Geophys. Res., 89, 9703

Holzer, T. E., \& Leer, E. 1980, J. Geophys. Res., 85, 4665

Huba, 1995, Phys. Plasmas, 2, 2502

Kohl et al. 1996, 31st Scientific Assembly of COSPAR, Birmigham, England

Kopp, R. A., \& Holzer, T. E. 1976, Sol. Phys., 49, 43

Miteeva, R., Zhelyazkov, I., \& Erdélyi, R. 2003, Phys. Plasmas, in press

Mjølhus, E. 1976, J. Plasma Phys., 16, 321

Mjølhus, E., \& Hada, T. 1997, in Nonlinear Waves and Chaos in Space Plasmas, ed. T. Hada, \& H. Matsumoto (TERRAPUB, Tokyo), 121

Nakariakov, V. M., \& Roberts, B., Phys. Lett. A, 254, 314

Noci, G., Kohl, J. L., Antonucci, E., Tondello, G., \& Huber, M. C. E. 1996, 31st Scientific Assembly of COSPAR, Birmigham, England

Ofman, L., \& Davila, J. M. 1995, J. Geophys. Res., 100, 23413

Ofman, L., \& Davila, J. M. 1997a, ApJ, 476, 357

Ofman, L., \& Davila, J. M. 1997b, ApJ, 476, L51

Ofman, L., Romali, M., Polleto, G., Noci, G., \& Kohl, J. L. 2000, ApJ, 529, 592

Parker, E. N. 1958, ApJ, 128, 664

Philips et al. 1995, Geophys. Res. Lett., 22, 3301

Roberts, B., \& Mangeney, A. 1982, MNRAS, 198, 7P

Roberts, B. 1985, Phys. Fluids, 28, 3280

Roberts, B. 1987, ApJ, 318, 590

Ruderman, M. S. 1987, Fluid Dyn., 22, 299

Ruderman, M. S. 2002, J. Plasma Phys., 67, 271

Smith, E. J., Balogh, A., Neugebauer, M., \& McComas, D. 1995, Geophys. Res. Lett., 22, 3381

Spangler, S. R., \& Sheerin, J. P. 1982, ApJ, 255, 855

Spangler, S. R. 1985, ApJ, 299, 122

Zhelyazkov, I., Debosscher, A., \& Goossens, M. 1996, Phys. Plasmas, 3,4346

Zhelyazkov, I., \& Mann, G. 1999, Phys. Plasmas, 6, 2340

Woch, J., Axford, W. I., Mall, U., et al. 1997, Geophys. Res. Lett., 24, 2885

Wurz, P., \& Gabriel, A. H. 1999, in 8th SoHO Workshop, ed. J. C. Vial, \& B. Kaldeich-Schürmann, ESA SP-46, 87 